Citation: O'Brien, M., dasNair, R., \& Lincoln, N.B. (2012). A

comparison of the content of memory rehabilitation groups for patients with neurological disabilities. Neuropsychological Rehabilitation, 23(3):321-32. doi: 10.1080/09602011.2012.753920

\title{
A comparison of the content of memory rehabilitation groups for patients with neurological disabilities
}

\author{
Marie Claire O'Brien, Roshan das Nair, and \\ Nadina B Lincoln \\ Institute of Work, Health \& Organisations, University of Nottingham, \\ Nottingham, NG8 1BB
}

The aim of the study was to compare the fidelity of manualised group memory rehabilitation programmes for participants with neurological disabilities. A sample of 11 neurological patients with memory problems, enrolled in a randomised controlled trial comparing compensation, restitution and self-help treatments, were observed during group sessions. Time-sampling was used to record the activity of the participants and the content of the discussion at one minute intervals. There was a significant difference between groups in the amount of time the group leader and participants spent talking $(\boldsymbol{p}<.001)$. Participants in the compensation and restitution groups spent significantly more time in memory rehabilitation discussion than participants in the selfhelp group $(\boldsymbol{p}<.001)$. There was also a significant difference between the amount of time spent discussing internal and external memory aids in the compensation and restitution groups $(\boldsymbol{p}<.001)$. These results support the fidelity of the interventions provided. This study also highlights the usefulness of time-sampling as a method to record the content and activity in rehabilitation groups.

Keywords: Memory rehabilitation; Effectiveness; Time-sampling; Treatment fidelity; Brain damage. 
Although many studies have evaluated memory rehabilitation for people with neurological conditions, there is currently little detailed information available about the content of memory rehabilitation programmes. There are two main neuropsychological approaches to memory rehabilitation: restitution and compensation (das Nair \& Lincoln, 2012; Evans, 2006). Restitution-oriented memory rehabilitation techniques include repetitive practice drills, which aim to achieve memory benefits by targeting a person's underlying memory impairment. In contrast, compensatory approaches involve teaching patients with memory problems to bypass problems using external and internal memory aids (Wilson, 2000). These approaches guide the content of the intervention.

In addition, rehabilitation for memory deficits may be delivered individually or in groups. Using a group format for memory rehabilitation has been suggested as both cost-efficient and more enjoyable for patients (Tate, 1997). There are studies to suggest that group interventions may improve outcomes for patients with memory problems. Some studies focus on teaching compensatory strategies in groups. For example, Schmitter-Edgecombe and Fahy (1995) trained eight patients with head injury and mild memory impairments to use a memory notebook. Patients in the intervention group reported significantly fewer everyday memory problems after treatment, compared to eight patients who received supportive therapy. There were no significant differences on neuropsychological assessments. However, only one patient was functioning at an impaired level of memory ability, as assessed on the Wechsler Memory Scale - Revised (WMS-R). Evans and Wilson (1992) found that five patients with brain injuries who attended a memory group for 11 months showed significantly increased levels of memory aids and strategy use and decreased levels of anxiety and depression. Patients did not show improvements on the Rivermead Behavioural Memory Test (RBMT). However, because this study lacked a control group, it could not be determined whether participants were simply benefiting from the social interaction.

Other group interventions involve training people to use a combination of internal and external aids. For example, Jennett and Lincoln (1991) used a cross-over design to investigate the effectiveness of teaching 20 stroke and head injury patients to use both internal and external strategies. No significant treatment effect was found. However, the number of reported memory aids used by patients after attending the group increased and the number of items reported as troubling patients on a subjective memory questionnaire decreased. The authors suggested that the failure to find statistically significant results may be due to the small sample size. 
Few studies have compared different types of group intervention. Hildebrandt, Bussmann-Mork, and Schwendemann (2006) investigated the effectiveness of group therapy for patients of mixed aetiology with "moderately" impaired memory. They compared process-oriented treatment, which concentrated on acquisition and recall strategies ( $n$ 1/4 24), strategy training, which included education and some compensatory memory training (n $1 / 4$ 22), and a control treatment (n $1 / 4$ 16), which involved low-dose memory training. The results demonstrated that over a five week period of intensive training only the group that received training in acquisition and recall strategies exhibited a significant improvement in comparison to the control group. However, as the authors acknowledged, this study was limited by failing to incorporate blind outcome assessors.

Reviews of the effectiveness of memory rehabilitation after brain damage have not separately evaluated individual and group therapies. However, they suggest there may be benefits of rehabilitation programmes for people with memory problems. In a review of the effectiveness of cognitive rehabilitation for traumatic brain injury and stroke, Cicerone et al. (2011) indicated that there was evidence to support the use of memory training using internal and external aids for patients with mild memory impairments. They also recommended that those with moderate to severe problems receive training in the use of external compensation strategies with direct application to functional activities. However, a recent Cochrane Review concluded that there was no evidence to support the effectiveness of memory rehabilitation for people with multiple sclerosis (das Nair et al., 2012). The authors argued that this finding could have been due to the limited quality of the studies reviewed, and made a call for more robust randomised trials to determine effectiveness. In addition, a systematic review of the content of cognitive rehabilitation programmes for people with acquired brain injury (Van Heughten, Gregorio, \& Wade, 2012) highlighted the lack of detail provided in most studies about the content of intervention.

das Nair and Lincoln (2012) conducted a single-blind randomised controlled trial in which participants with memory problems were allocated to different types of group memory rehabilitation programmes. Treatment was based on a manual developed for the study (das Nair, 2007). The compensation group received training in the use of external and internal compensatory memory aids. The restitution group mainly received training in the use of internal aids, in addition to attention training and training in encoding strategies. The control group was a self-help group in which participants were encouraged to discuss emotional issues related to their memory impairments and taught relaxation techniques. There were no significant differences in outcomes, as assessed on measures of self-reported memory problems in daily life, mood, adjustment or activities of daily living. There were significant differences between the groups in the use of memory aids. One possible 
explanation for the lack of treatment effect is that there may have been overlap in the content of the interventions between the three groups. This could have masked any benefits of the specific intervention provided. It was therefore necessary to determine the fidelity of the intervention.

Fidelity is the first stage of process evaluation (Saunders, Evans, \& Joshi, 2005) and is designed to determine the extent to which intervention was implemented as planned. Although all three groups were run according to a manual, it was important that observations of the actual running of memory rehabilitation groups were undertaken to document the fidelity of the intervention. Time-sampling has been previously used as a method of collecting systematic observations, (Lincoln, Willis, Phillips, Juby, \& Berman, 1996). The aim of this study was to observe memory rehabilitation groups as part of the evaluation conducted by das Nair and Lincoln (2012) in order to document the fidelity of the intervention provided. At the time the observations were conducted the outcome of the trial was not known.

\section{METHOD}

Ethical approval was by granted by Nottingham Research Ethics Committee 1 .

Participants were recruited from a randomised trial (das Nair \& Lincoln, 2012) of rehabilitation of memory for people with neurological disabilities. In this trial, participants with memory problems were recruited through referrals from health professionals working in hospitals and the community, and posters advertising the study. They had memory problems following a neurological disability (stroke, multiple sclerosis, traumatic brain injury), were 18 years of age and older, living within a 50 mile radius and had been diagnosed at least one month prior to recruitment. Participants were excluded if they were blind or deaf, had a previous diagnosis of brain damage, had a current diagnosis of mental health problems, or did not speak English. Participants for the present study were those attending group sessions during the period when an independent observer was available.

Participants recruited to the trial were randomly allocated to one of three types of intervention group (compensation or restitution memory rehabilitation groups or a self-help control group). Once participants were allocated to a group, the group leader held two individual sessions with each participant to determine specific memory problems experienced, to provide information about the trial, and to arrange group sessions. Four participants were invited to each group session. Participants received 10 group sessions, each lasting approximately an hour and a half. The groups were all run by assistant psychologists supervised by one clinical psychologist (RDN), experienced in neuropsychological rehabilitation. There was a manual for each type of group documenting topics to be covered in each session and strategies to 
advise. An observer (the first author; MO), independent to the trial, attended group sessions and recorded the activity and content of the sessions on the minute every minute. An attempt was made by the observer to attend all 10 sessions for each group in order to ensure data collected were representative of the whole programme and to reduce reactive effects within the groups (Bowling, 2002). A stopwatch was used by the observer to keep time.

Observations were made in two categories (activity and content) for the group leader and each participant. There were two activity categories; talking to the group or listening to a group member. There were eight content categories, comprising activities and topics likely to occur in any of the group sessions, based on information contained in the manual. The content categories included components of rehabilitation; memory theories (e.g., "The working memory is. . ."), memory processes (e.g., "The importance of paying attention. . ."), internal aids ("chunking"), and external aids (e.g., "look at the memory board"). The "non-rehabilitation content" categories included talk about forgetting (e.g., ". . . forgot to buy milk") which did not involve consideration of how to cope with the problem, relaxation exercises (e.g., “. . .keep arms loose and light. .."), group-related issues (e.g., “. . .the plan for the next session. . ."), illness-related issues (e.g., ". . . diagnosed two years ago"), social activities / lifestyle (e.g., "went for a walk"), and emotion and feelings (e.g., ". . . feel content"). The content of each observation was recorded, in situ, qualitatively. For example, a few exact words of the person speaking, such as "the aim of the group is to reduce memory problems", that represented the topic being talked about at that time, were written down (also see examples above). The observer also documented which group member was talking. These observations were then categorised according to their content. In order to ensure consistency in the coding of the content observations, a second rater, blind to the original coding, also classified the observations into content categories.

Results were analysed using SPSS version 16. Chi square tests were used to compare the frequencies of activity in each type of memory rehabilitation group.

\section{RESULTS}

The activity and content of the groups was observed in seven compensation (502 minutes), eight restitution (408 minutes), and nine self-help (458 minutes) group sessions; with four, three, and four participants, respectively. The characteristics of participants are shown in Table 1.

Inter-rater agreement of the content categories was checked by calculating the level of agreement between the raters. The level of agreement was $97 \%$. The level of agreement on each category of content is shown in Table 2. 
TABLE 1

Characteristics of the participants observed

\begin{tabular}{|c|c|c|c|c|c|}
\hline \multirow[b]{2}{*}{ Characteristic } & & \multicolumn{3}{|c|}{ Intervention group } & \multirow{2}{*}{$\frac{\text { Comparison }}{p}$} \\
\hline & & $\begin{array}{c}\text { Compensation } \\
n=4\end{array}$ & $\begin{array}{c}\text { Restitution } \\
n=3\end{array}$ & $\begin{array}{c}\text { Self-help } \\
n=4\end{array}$ & \\
\hline Gender & $\begin{array}{l}\text { Men } \\
\text { Women }\end{array}$ & $\begin{array}{l}0 \\
4\end{array}$ & $\begin{array}{l}2 \\
1\end{array}$ & $\begin{array}{l}4 \\
0\end{array}$ & $.27^{++}$ \\
\hline Aetiology & $\begin{array}{l}\text { MS } \\
\text { Stroke }\end{array}$ & $\begin{array}{l}4 \\
0\end{array}$ & $\begin{array}{l}2 \\
1\end{array}$ & $\begin{array}{l}4 \\
0\end{array}$ & $.23^{++}$ \\
\hline Age & $\begin{array}{l}\text { Median } \\
\text { IQR }\end{array}$ & $\begin{array}{c}41.0 \\
40.0-43.0\end{array}$ & $\begin{array}{c}49.0 \\
40.0-50.0\end{array}$ & $\begin{array}{c}56.5 \\
37.0 \mathrm{v} 62.0\end{array}$ & $.36^{+}$ \\
\hline Months since onset & $\begin{array}{l}\text { Median } \\
\text { IQR }\end{array}$ & $\begin{array}{l}7.0 \\
6-84\end{array}$ & $\begin{array}{l}17.0 \\
15-113\end{array}$ & $\begin{array}{c}60.0 \\
24-114\end{array}$ & $.33^{+}$ \\
\hline NART Estimated IQ & $\begin{array}{l}\text { Median } \\
\text { IQR }\end{array}$ & $\begin{array}{c}107.0 \\
96.3-116.3\end{array}$ & $\begin{array}{c}101.0 \\
97.0-103.0\end{array}$ & $\begin{array}{c}106.5 \\
99.0-108.0\end{array}$ & $.47^{+}$ \\
\hline RBMT-E Profile score & $\begin{array}{l}\text { Median } \\
\text { IQR }\end{array}$ & $\begin{array}{c}2.0 \\
1.3-2.0\end{array}$ & $\begin{array}{l}1.0 \\
1.0-2.0\end{array}$ & $\begin{array}{c}0.5 \\
0.0-1.8\end{array}$ & $.23^{+}$ \\
\hline EMQ Total & $\begin{array}{l}\text { Median } \\
\text { IQR }\end{array}$ & $\begin{array}{c}36.0 \\
29.0-76.0\end{array}$ & $\begin{array}{c}68.0 \\
24.0-82.0\end{array}$ & $\begin{array}{c}61.0 \\
41.8-72.8\end{array}$ & $.85^{+}$ \\
\hline GHQ12 & $\begin{array}{l}\text { Median } \\
\text { IQR }\end{array}$ & $\begin{array}{c}4.5 \\
3.3-10.3\end{array}$ & $\begin{array}{c}7.0 \\
5.0-10.0\end{array}$ & $\begin{array}{c}6.5 \\
1.5-10.8\end{array}$ & $.82^{+}$ \\
\hline NEADL & $\begin{array}{l}\text { Median } \\
\text { IQR }\end{array}$ & $\begin{array}{c}49.5 \\
39.0-55.5\end{array}$ & $\begin{array}{c}49.0 \\
36.0-60.0\end{array}$ & $\begin{array}{c}47.5 \\
33.8-62.7\end{array}$ & $.98^{+}$ \\
\hline SSTALD & $\begin{array}{l}\text { Median } \\
\text { IQR }\end{array}$ & $\begin{array}{c}20.0 \\
20.0-20.0\end{array}$ & $\begin{array}{c}18.0 \\
16.0-20.0\end{array}$ & $\begin{array}{c}18.5 \\
15.8-19.0\end{array}$ & $.12^{+}$ \\
\hline
\end{tabular}

NART $=$ National Adult Reading Test; RBMT-E $=$ Rivermead Behavioural Memory Test-Extended Version; EMQ $=$ Everyday Memory Questionnaire; GHQ-12 = General Health Questionnaire-12; NEADL = Nottingham Extended Activities of Daily Living scale; SSTALD = Sheffield Screening Test for Acquired Language Disorders; MS = Multiple Sclerosis; IQR = Interquartile range.

+ Kruskal Wallis test; ++ Chi Square test. 
TABLE 2

Inter-rater reliability of observations in group sessions

\begin{tabular}{lrrr}
\hline Number of observations & Rater 1 & Rater 2 & Discrepancy \\
\hline Rehabilitation discussion & & & \\
External aids & 156 & 153 & 3 \\
Internal aids & 360 & 371 & 11 \\
Memory theory & 41 & 41 & 0 \\
Memory processes & 114 & 110 & 4 \\
Non-rehabilitation discussion & & & \\
Memory/forgetting issues & 138 & 144 & 6 \\
Relaxation exercise & 172 & 168 & 4 \\
Group-related issues & 83 & 89 & 6 \\
Social activities & 114 & 110 & 4 \\
Emotions and feelings & 47 & 48 & 1 \\
Illness related & 143 & 144 & 40 \\
Total & 1368 & 1386 & \\
\hline
\end{tabular}

TABLE 3

Distribution of activity and content in group sessions

\begin{tabular}{|c|c|c|c|c|}
\hline & & \multicolumn{3}{|c|}{ Intervention groups } \\
\hline & & Compensation & Restitution & Self-help \\
\hline & Observations & 502 & 408 & 458 \\
\hline & Participants & 4 & 3 & 4 \\
\hline \multicolumn{5}{|l|}{ Activity } \\
\hline \multirow{2}{*}{ Time talking* } & Group leader & 47.6 & 63.0 & 56.8 \\
\hline & Participants & 52.4 & 37.0 & 43.2 \\
\hline \multicolumn{5}{|l|}{ Content } \\
\hline \multicolumn{5}{|l|}{ Rehabilitation discussion } \\
\hline External aids & & 26.5 & 5.6 & 0.0 \\
\hline Internal aids & & 29.9 & 51.2 & 0.2 \\
\hline Memory theory & & 1.8 & 1.7 & 5.5 \\
\hline Memory processes & & 12.5 & 10.0 & 2.2 \\
\hline \multicolumn{5}{|l|}{ Non-rehabilitation discussion } \\
\hline Memory/forgetting issues & & 8.8 & 18.4 & 4.1 \\
\hline Relaxation exercise & & 0.0 & 0.0 & 37.6 \\
\hline Group-related issues & & 3.4 & 6.9 & 8.3 \\
\hline Social activities & & 8.2 & 4.4 & 12.0 \\
\hline Emotions and feelings & & 2.6 & 0.0 & 7.4 \\
\hline Illness related & & 6.4 & 1.7 & 22.7 \\
\hline Total rehabilitation & & 70.7 & 68.5 & 7.9 \\
\hline Total non-rehabilitation & & 29.4 & 31.4 & 92.1 \\
\hline
\end{tabular}

* In minutes. 
The results of the observations of whether participants were talking or listening are shown in Table 3. There was a significant difference between the proportions of time the group leader and participants spent talking between the groups $\left(x^{2}=22.17, d f=2, p<.001\right)$. The group leader spent more time talking, and the participants less time talking, in the restitution group compared to the compensation group.

The proportion of observations in each content category is also summarised in Table 3. Participants in the compensation group spent more time discussing external memory aids $(26.5 \%)$ than participants in the restitution group (5.6\%). External memory aids were not discussed in the self-help group. Internal memory aids were discussed most in the restitution group (51.2\%) in comparison to the compensation $(29.9 \%)$ and self-help groups $(0.2 \%)$. The self-help group spent less time discussing memory processes $(2.2 \%)$ compared to the intervention groups $(12.5 \%$ and $10 \%)$. The relaxation exercise was only discussed and implemented in the self-help group (37.6\%). Overall, the proportion of time spent in memory rehabilitation discussion, was high for the compensation (70.7\%) and restitution groups $(68.5 \%)$ in contrast to the self-help control group (7.9\%).

There was a significant difference between the time spent in memory rehabilitation and non-memory rehabilitation discussions between the compensation, restitution and self-help groups $\left(\mathrm{x}^{2}=467.8, d f=2, p<.001\right)$. Members of the compensation group spent significantly more time engaging in memory rehabilitation talk, in comparison to non-memory rehabilitation talk, than the self-help group $\left(\mathrm{x}^{2}=391.9, d f=1, p<.001\right)$. People in the restitution group also spent significantly more time in memory rehabilitation discussion in contrast to non-memory rehabilitation discussion, than the selfhelp group $\left(x^{2}=343.8, d f=1, p<.001\right)$. However, there was no statistically significant difference in time spent in memory rehabilitation and nonmemory rehabilitation discussions between the compensation and restitution groups $\left(\mathrm{x}^{2}=466.0, d f=1, p=.50\right)$. There was also a significant difference between the amount of time spent discussing internal and external memory aids in the compensation and restitution groups $\left(\mathrm{x}^{2}=83.0, d f=1, p<\right.$ $.001)$.

In the compensation group, external aids discussed included: diaries, notice boards, white boards, wall charts, lists, Post-its, calendars, writing down information, leaving things in a strategic place, mobile phones, and alarms. Internal aids discussed in the compensation group, included attention to detail, active observation, deep level processing, the make-a-story method, rhymes, the PQRST method, asking "when, where, who, how and why" questions, first letter cues, breaking down tasks into smaller units, association, rehearsal, imagery, categorisation, and errorless learning. Other memory rehabilitation topics that were discussed in the compensation group included 
memory processes, and to a lesser extent, memory theories. Attention, as opposed to encoding, storage or retrieval was the main memory process discussed in the compensation group.

Memory rehabilitation content in the restitution group, included discussion of the following internal aids: rehearsal, association, mental maps, chunking, attention to detail, visual imagery, asking "when, where, who, how and why" questions, categorisation, deep level processing, first letter cues, rhyming, the make-a-story method and the PQRST method. Some external aids mentioned included: writing down information, calendars, diaries, and mobile phones, but occurred less frequently than in the compensation group. Other memory rehabilitation content that was documented in the restitution group included memory processes. Attention was also the most frequently discussed memory process in the restitution group.

\section{DISCUSSION}

The main finding was that the compensation and restitution groups received a similar amount of memory rehabilitation and the self-help group significantly less. This indicates that the lack of significant differences in outcome between the groups (das Nair \& Lincoln, 2012) was not due to the self-help group receiving a comparable amount of memory rehabilitation. It supports the fidelity of the intervention provided. In addition, there was a significant difference in the amount of time spent discussing internal and external memory aids in the compensation and restitution groups. These findings indicate that the groups were run in accordance with the manual. It also means that the lack of any statistically significant differences in outcome cannot be attributed to the failure to deliver the groups as intended. However, there was some overlap in content between the two interventions.

The group leader and participants spent significantly different amounts of time talking in each type of group. The group leader probably spent more time talking in the self-help group because this group was mainly engaged in the relaxation exercise, which was directed by the group leader. The group leader also spent more time talking in the restitution group than the compensation group. This may be because there were three participants in the group, whereas there were four participants in the compensation group. Additionally, one of the participants in the restitution group had an expressive language disorder, so did not speak as much as other participants, and thus may have required the group leader to talk more.

The memory rehabilitation content in the compensation group covered a range of external and internal strategies and using errorless learning. In contrast, memory rehabilitation content in the restitution group mainly included discussion of internal aids. Although errorless learning was not documented 
as having been discussed in the restitution group, it may have been discussed in one of the group sessions the observer did not attend. In addition, some external aids were mentioned in the restitution group, but such talk was generated by the participants and not the group leader. Therefore, the memory rehabilitation content in the restitution group was dominated by discussion of internal strategies and the value of attention in supporting memory.

These findings support the differences between the groups. However, the overlap also suggests that both types of strategies need to be discussed to some extent. In clinical practice both compensation and restitution strategies are used together and therefore, for further clinical evaluations of the interventions, these strategies might be delivered as a combined intervention.

In both intervention groups most time was devoted to discussing daily problems in memory that participants encountered, such as forgetting to buy groceries; group-related issues, such as homework; social activities, such as plans for the week; emotions and feelings; and illness-related issues, such as fatigue. Similar to previous research that highlighted the social and emotional benefits of neuropsychological rehabilitation groups (Evans \& Wilson, 1992; Tate, 1997), non-treatment related topics emerged and were discussed. Topics discussed in the self-help group included the same issues, but these occurred more frequently than in the memory rehabilitation groups.

There are limitations to this study. Despite the fact that the observer attended most group sessions to minimise reaction effects exhibited by patients within the groups (Bowling, 2002), the presence of the observer may have affected how the group leader ran the groups and what was discussed. Additionally, it was not considered appropriate to have a second observer in group sessions in order to calculate inter-observer reliability of the observations directly. Consequently, observations may have been biased by expectations about the differences between the groups. However, by recording observations qualitatively as opposed to categorising observations immediately, and by calculating inter-rater reliability using the recorded observations, observer bias may have been minimised.

Using one-minute recording intervals increased the likelihood that results were representative of what happened in the groups, even though some information may have been missed. Some topics, such as errorless learning, may have been discussed but were not documented because they occurred in groups the observer did not attend. Nevertheless, systematic observations enabled a more accurate comparison of activity and content in the memory groups than would have been derived from interviews or retrospective questioning. In addition, there were more observations made during compensation groups and fewer during restitution groups so that more information may have been missed during restitution groups.

Information about activities may be limited by the inclusion of only two activity categories in the time sampling schedule. However, given that the 
groups were small and relatively short, there was generally one person speaking in the group at any time. Consequently, the other participants were considered to be listening even though they may have been doing other things at the same time, such as writing notes or drinking tea. Although the observer could never be completely sure whether the group members were actually listening to the speaker, it is suggested that in such small groups where the speakers frequently directed attention to all members, it would have been difficult not to listen most of the time.

Only 11 participants were observed in this study and it is possible the intervention with them may have differed from that given to other participants. However, the characteristics of this sample are similar to those in the trial (das Nair \& Lincoln, 2012), with the majority having multiple sclerosis, and the average age in the late 40s. However, in this study there were more men than women $(55 \%)$ in contrast to slightly more women (56\%) in the main study, but the differences are unlikely to have produced any bias in the results and there is no evidence to suggest that interventions vary according to the gender of the participants.

Overall, the time-sampling data supported the results of the randomised trial by confirming the interventions were delivered as intended and that any differences in outcome can be attributed to different content in the three types of groups. The observations were analysed before the outcome of the trial was known and so were unlikely to have been biased by knowledge of the results. This study revealed that rehabilitation content was high in memory rehabilitation groups and there was very little memory rehabilitation content in the control group. This supports the treatment fidelity of the interventions provided and indicates that the self-help group controlled for attention without providing memory rehabilitation. This study also demonstrates that observations of complex interventions in randomised trials can be done with minimal effect on the group facilitator or participants, and with scientific rigour. Such observations improve the trustworthiness of the interpretation of randomised trials of complex interventions.

\section{REFERENCES}

Bowling, A. (2002). Research methods in health. New York: Open University Press.

Cicerone, K. D., Langenbahn, D. M., Braden, C., Malec, J. F., Kalmar, K., Fraas, M., ... Ashman, T. (2011). Evidence based cognitive rehabilitation: Updated review of the literature from 2003 through 2008. Archives of Physical Medicine \& Rehabilitation, 92, $519-530$.

das Nair, R. (2007). Effectiveness of memory rehabilitation following brain damage: A single blind randomised controlled trial. $\mathrm{PhD}$ Thesis, University of Nottingham. 
das Nair, R., Ferguson, H., Stark, D., \& Lincoln, N. (2012). Memory rehabilitation for people with multiple sclerosis. Cochrane Database of Systematic Reviews. Issue 3, Art. No.: CD008754. DOI: 10.1002/14651858.CD008754.pub2.

das Nair, R., \& Lincoln, N. B. (2012). Evaluation of memory rehabilitation in people with neurological disabilities: A randomised controlled trial (REMIND). Clinical Rehabilitation, 26, 894-903.

Evans, J. J. (2006). Memory rehabilitation - should we be aiming for restoration or compensation? Journal of Neurology, 253, 520-521.

Evans, J. J., \& Wilson, B. A. (1992). A memory group for individuals with brain injury. Clinical Rehabilitation, 6, 75-81.

Hildebrandt, H., Bussmann-Mork, B., \& Swendeman, G. (2006). Group therapy for memory impaired patients: A partial remediation is possible. Journal of Neurology, 253, 512-519.

Jennett, S. M., \& Lincoln, N. B. (1991). An evaluation of the effectiveness of group therapy for memory problems. International Disability Studies, 13, 83-86.

Lincoln, N. B., Willis, B. A., Phillips, M., Juby, L. C., \& Berman, P. (1996). Comparison of rehabilitation practice on hospital wards for stroke patients. Stroke, 27, 18-23.

Saunders, R. P., Evans, M. H., \& Joshi, P. (2005). Developing a process-evaluation plan for assessing health promotion program implementation: A how-to guide. Health Promotion Practice, 6, 134-147.

Schmitter-Edgecombe, M., \& Fahy, J. F. (1995). Memory remediation after severe closed head injury: Notebook training versus supportive therapy. Journal of Consulting and Clinical Psychology, 63, 484-489.

Tate, R. L. (1997). Beyond one-bun, two shoe: Recent advances in the psychological rehabilitation of memory disorders after acquired brain injury. Brain Injury, 11, 907-918.

Van Heughten, C., Gregorio, G. W., \& Wade, D. T. (2012). Evidence-based cognitive rehabilitation after acquired brain injury: A systematic review of the content of treatment. Neuropsychological Rehabilitation, 22, 653-673.

Wilson, B. A. (2000). Compensating for cognitive deficits following brain injury. Neuropsychology Review, 10, 233-243. 